\title{
Jagged 2 silencing inhibits motility and invasiveness of colorectal cancer cell lines
}

\author{
WAN HE ${ }^{1}$, CHARLES MING LOK CHAN ${ }^{2}$, SZE CHUEN CESAR WONG $^{3}$, \\ THOMAS CHI CHUEN AU ${ }^{2}$, WING SHAN HO${ }^{2}$, AMANDA KIT CHING CHAN ${ }^{4}$, \\ ANDREW SAI KIT CHAN ${ }^{2}$, BRIGETTE BUIG YUE MA ${ }^{2}$ and ANTHONY TAK CHEUNG CHAN ${ }^{2}$ \\ ${ }^{1}$ Department of Oncology, Shenzhen People's Hospital, Second Clinical Medical College of Jinan University, \\ Shenzhen, Guangdong 518029; ${ }^{2}$ State Key Laboratory of Oncology in South China, Sir YK Pao Center for Cancer, \\ Department of Clinical Oncology, Hong Kong Cancer Institute and Prince of Wales Hospital, \\ The Chinese University of Hong Kong; ${ }^{3}$ Department of Health Technology and Informatics, Hong Kong \\ Polytechnic University; ${ }^{4}$ Department of Pathology, Queen Elizabeth Hospital, Hong Kong SAR, P.R. China
}

Received December 19, 2014; Accepted June 27, 2016

DOI: $10.3892 / 01.2016 .5321$

\begin{abstract}
Although the Notch pathway has been reported to be activated in colorectal cancer (CRC), limited information is available regarding the expression and role of its ligand, Jagged 2 (JAG2), in CRC. Using immunohistochemistry, the present study demonstrated that JAG2 protein expression may be detected in up to $95 \%$ of CRC cases and is 3 -fold upregulated in tumor cells compared to surrounding normal tissues. This finding suggests that JAG2 may have a role in the tumorigenicity of CRC. To further investigate the cellular functions of JAG2 expression in CRC, two different small interfering RNAs (siRNAs) were used to downregulate JAG2 expression in CRC cell lines (HCT116, DLD-1 and HT-29). The results indicated that JAG2 knockdown inhibits the motility and invasiveness of CRC cell lines without significantly affecting cell proliferation. These findings implicate JAG2 in promoting aggressiveness of CRC, and lay the foundation for its future development as a therapeutic target for the treatment of CRC.
\end{abstract}

Correspondence to: Professor Anthony Tak Cheung Chan, State Key Laboratory of Oncology in South China, Sir YK Pao Center for Cancer, Department of Clinical Oncology, Hong Kong Cancer Institute and Prince of Wales Hospital, The Chinese University of Hong Kong, 30-32 Ngan Shing Street, Shatin, New Territories, Hong Kong SAR, P.R. China

E-mail: anthony@clo.cuhk.edu.hk

Professor Sze Chuen Cesar Wong, Department of Health Technology and Informatics, Hong Kong Polytechnic University, Room Y934, 9/F, Lee Shau Kee Building, Hung Hom, Kowloon, Hong Kong SAR, P.R. China

E-mail: cesar.wong@polyu.edu.hk

Abbreviations: CRC, colorectal cancer; IHC, immunohistochemical; JAG2, Jagged 2; siRNA, small interfering RNA

Key words: Notch, Jagged 2, colorectal cancer, migration, invasion

\section{Introduction}

Colorectal cancer (CRC) is the third most common cancer in males and the second in females, respectively, with $>1.4$ million newly diagnosed cases and 693,933 mortalities estimated to have occurred in 2012, accounting for $8.5 \%$ of all cancer mortalities, thus making CRC the fourth most common cause of mortality from cancer worldwide (1). Approximately $20 \%$ of CRC patients present with metastases at the time of diagnosis, and $\sim 50 \%$ of the patients without metastases at presentation exhibit distant metastases within 3 years of diagnosis (2). For patients with unresectable metastatic CRC, prognosis is poor, with a 5 -year survival of $<10 \%$; however, a marked benefit in median overall survival may be achieved with palliative systemic therapy (3). Significant advances in systemic treatment for metastatic CRC, including targeted therapies, have improved survival; however, even with combination of target agents and chemotherapy, the median survival of CRC patients is only 29 months (4). A better understanding of the factors that lead to tumor progression and metastasis is urgently required for the development of novel strategies for CRC treatment.

The Notch pathway is highly conserved and functions in numerous biological processes, including cell differentiation, proliferation and death (5-7). Mammals have four types of membrane-bound Notch receptors (Notch 1-4) and five types of membrane bound ligands (Jagged 1-2, and Delta-like 1, 3 and 4) (2). Upon ligand binding, Notch receptors undergo proteolytic cleavage to release the Notch intracellular domain, which enters the nucleus and associates with DNA binding proteins to act as a transcriptional factor for the regulation of gene transcription.

Notch pathway signaling is activated in several types of cancer, including CRC, T-cell acute lymphoblastic leukemia (T-ALL) (8) and breast cancer (9). Among the Notch ligands, upregulation of JAG2 expression has been shown to be significantly associated with vascular development, metastasis-free and overall survival in breast cancer patients $(10,11)$. In 
ovarian carcinoma, elevated JAG2 levels are reported to be associated with lymph node and distant metastases (12). These findings are indicative of a pivotal role for JAG2 expression during cancer progression. However, there is extremely limited information available with regard to the expression pattern and functional role of JAG2 protein in human CRC. Therefore, the present study aimed to investigate the expression and function of JAG2 in human CRC. JAG2 protein expression was assessed in 40 cases of human CRC tissues and 7 human colon cell lines, while its functions were studied by RNA interference in 3 CRC cell lines. The effects of JAG2 silencing on the cellular functions of CRC cell lines were assessed by wound healing assay, Matrigel invasion assay and cell growth assay.

\section{Materials and methods}

Cell lines and tissues. The human CRC cell lines SW480, SW620, HCT116, DLD-1, HT29 and RKO, and a normal colon cell line, CCD-18Co, were purchased from the American Type Culture Collection (ATCC; Manassas, VA, USA) and cultured in RPMI-1640 medium supplemented with $10 \%$ fetal bovine serum (Thermo Fisher Scientific, Inc., Waltham, MA, USA) at $37^{\circ} \mathrm{C}$ in a humidified incubator with $5 \% \mathrm{CO}_{2}$.

Formalin-fixed, paraffin-embedded colorectal carcinoma specimens were obtained from the Department of Pathology of Queen Elizabeth Hospital (Hong Kong SAR, China). Approval from an institutional ethics review board and informed consent from all participants were obtained.

Immunohistochemical (IHC) staining. Sections (4- $\mu \mathrm{m}$ thick) were de-waxed, rehydrated and stained for JAG2 protein expression using a rabbit anti-human JAG2 polyclonal antibody (\#06-1097; EMD Millipore, Billerica, MA, USA; dilution, 1:500) for $92 \mathrm{~min}$ at room temperature in a Ventana BenchMark XT processor (Ventana Medical Systems, Tucson, AZ, USA) and counterstained with hematoxylin. The negative control included sections incubated with antibody dilution buffer (\#950-300; Ventana Medical Systems) without primary antibody. Slides were visualized using the ultraView Universal DAB Detection kit (\#760-500; Ventana Medical Systems). Two independent observers who were blinded to the patients' clinical information assessed the scoring of positive staining signals. In each patient tissue specimen, 5 fields at x400 magnification were evaluated. The scoring of staining intensity was as follows: 0 , negative; 1 , weak; 2 , moderate; 3 , strong; and 4, very strong. An IHC score ranging from 0 to 400 was obtained by multiplying the percentage of the positive cells (0-100\%) by the staining intensity (score 0-4) (13).

Western blot. Cells were lysed in buffer containing sodium dodecyl sulfate, protease inhibitors and phosphatase inhibitors (Roche Diagnostics, Basel, Switzerland). Equal amounts of protein lysates were gel-separated and transferred onto nitrocellulose membranes (Bio-Rad Laboratories, Inc., Hercules, CA, USA). Blocking was conducted in a buffer containing 5\% non-fat milk and $0.05 \%$ Tween 20 in Tris-buffered saline for $1 \mathrm{~h}$ at room temperature. Primary antibodies were incubated at $4^{\circ} \mathrm{C}$ overnight, while secondary antibodies were incubated for $1 \mathrm{~h}$ at room temperature. Protein bands were detected with SuperSignal ${ }^{\circledR}$ West Pico Chemiluminescent Substrate (Thermo
Fisher Scientific, Inc.) and Hyperfilm ECL film (GE Healthcare, Uppsala, Sweden). The following antibodies were used: Rabbit anti-human JAG2 monoclonal antibody (\#2210; Cell Signaling Technology, Danvers, MA, USA; dilution, 1:1,000); mouse anti-actin monoclonal antibody (\#ab3280; Abcam, Cambridge, UK; dilution, 1:50,000); horseradish peroxidase (HRP)-conjugated goat anti-mouse IgG polyclonal secondary antibody (\#170-6516; Bio-Rad Laboratories, Inc.; dilution, 1:100,000); HRP-conjugated goat anti-rabbit IgG polyclonal secondary antibody (\#81-6120; Thermo Fisher Scientific, Inc.; dilution, 1:100,000).

siRNA transfection. Cells were transfected with $25 \mathrm{nM}$ siRNA using Invitrogen Lipofectamine 2000 (Thermo Fisher Scientific, Inc.). The siRNAs used included ON-TARGETplus Human JAG2 siRNA (GE Healthcare Dharmacon, Inc., Lafayette, CO, USA) (siRNA1), JAG2 siRNA (\#s7645; Thermo Fisher Scientific, Inc.) (siRNA2) and Silencer Select Negative Control No. 1 siRNA (Thermo Fisher Scientific, Inc.). An untransfected control was created by replacing siRNAs with Opti-MEM Reduced Serum Medium (Thermo Fisher Scientific, Inc.).

Cell proliferation assay. At $72 \mathrm{~h}$ post transfection, $10 \mu \mathrm{l}$ of MTS reagent [3-(4,5-dimethylthiazol-2-yl)-5-(3-carb oxymethoxyphenyl)-2-(4-sulfophenyl)-2H-tetrazolium; Promega, WI, USA] was added to $100 \mathrm{ml}$ of fresh culture medium in each well of a 96-well plate and incubated for $3 \mathrm{~h}$ at $37^{\circ} \mathrm{C}$. Optical density at $490 \mathrm{~nm}$ was measured using the VICTOR ${ }^{3}$ Multilabel Plate Reader 1420 (PerkinElmer, Inc., Waltham, MA, USA). The surviving percentage of cells was calculated by the following formula: Cell proliferation $\%=\left(\mathrm{OD}_{\text {test sample }} / \mathrm{OD}_{\text {control sample }}\right) \times 100 \%$

The control sample reading was obtained from wells containing untransfected cells. The reading was taken as the mean of four wells, and the results were expressed as mean \pm standard error.

Monolayer scratch wound healing assay. siRNA-transfected cells were seeded into culture plates, allowed to form a confluent monolayer and serum starved overnight. Wounds were created by scratching with sterile pipette tips. Fresh culture medium was replenished every $24 \mathrm{~h}$ (serum-free culture medium for DLD-1 and HT29; culture medium supplemented with $1 \%$ serum for HCT116 cells). Photographs were taken under a microscope and the cell-free area was measured using ImageJ analysis software version 1.43 (http://rsb.info.nih. gov/ij). Wound closure was calculated by the following equation and expressed as a percentage relative to the untreated control, which was taken to be $100 \%$ (14):

$$
\text { Wound closure } \%=\frac{\left[(\text { cell free area })_{0}-(\text { cell free area })_{t}\right]_{\text {experiment }}}{\left[(\text { cell free area })_{0}-(\text { cell free area })_{t}\right]_{\text {control }}} \times 100 \%
$$

'Experiment' represents experimental samples and 'control' represents untreated control samples. The results were expressed as the mean \pm standard error of the mean (SEM) and plotted in bar charts. All experiments were performed in triplicate.

Matrigel invasion assay. Cells were harvested at $48 \mathrm{~h}$ post transfection and transferred to the upper chamber of BioCoat 

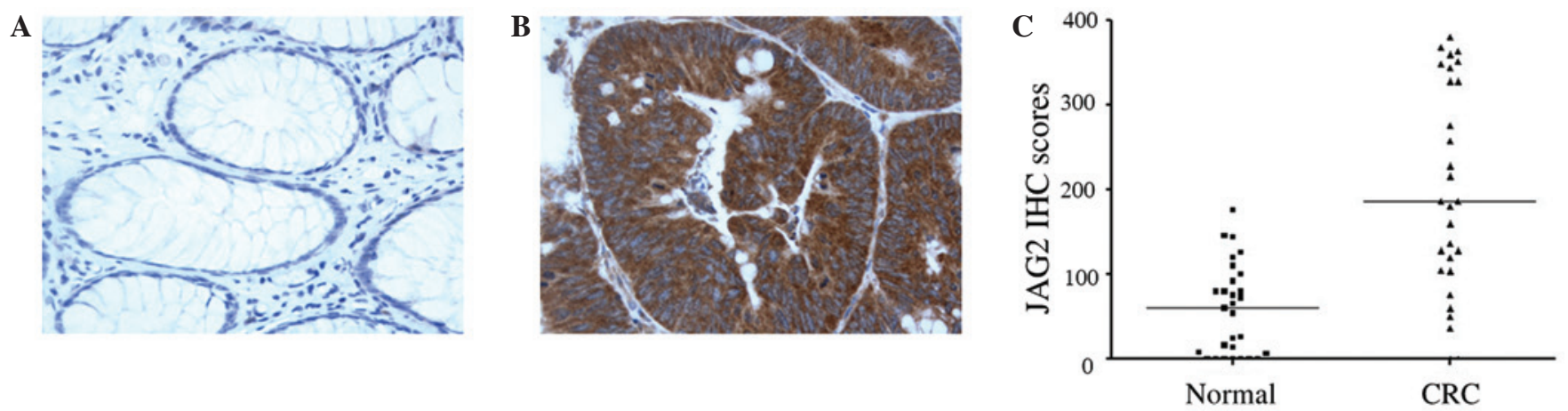

D Normal
cell line

CRC cell lines

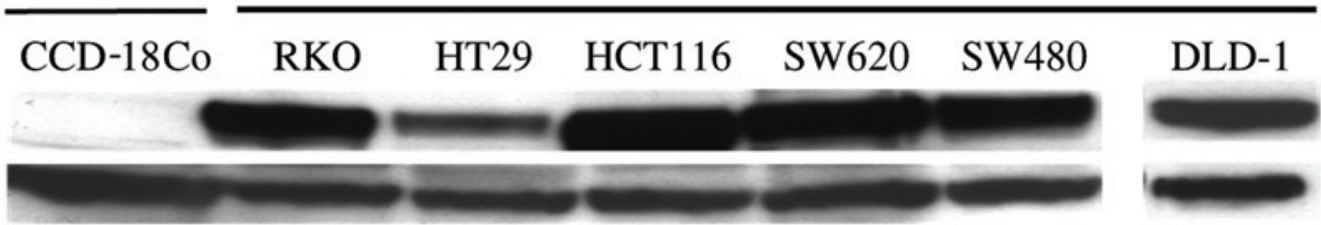

Figure 1. JAG2 protein expression in (A) normal colon and (B) CRC tissue as detected by IHC; brown staining indicates JAG2 while blue staining indicates the location of cell nucleus. (C) IHC scores for JAG2 expression in normal and colorectal cancer tissues; JAG2 protein expression was upregulated in CRC tissues compared to normal colon tissues. The horizontal line represents the median score for each category. (D) Detection of JAG2 protein expression by western blotting in a normal colon cell line and in 6 CRC cell lines; JAG2 protein expression is detected in CRC cell lines but not in the normal colon epithelial cell line, CCD-18Co. JAG2, Jagged 2; CRC, colorectal cancer; IHC, immunohistochemistry.

Matrigel Invasion Chambers (BD Biosciences, San Jose, CA, USA) in serum-free medium. Culture medium with $10 \%$ fetal bovine serum was added to the lower compartment as a chemoattractant. Following a 48 -h incubation at $37^{\circ} \mathrm{C}$ in a humidified incubator with $5 \% \mathrm{CO}_{2}$, cells remaining in the upper chamber were removed by cotton swabs and cells that had invaded to the bottom surface of the membrane were fixed in methanol and stained with $0.1 \%$ Toluidine Blue O (Sigma-Aldrich). The numbers of invaded cells on the bottom surface of the membrane were counted, expressed as mean \pm SEM and plotted in the bar charts. All experiments were repeated three times.

Statistical analysis. The differences in IHC scores between tumor tissues and normal colorectal epithelia were analyzed by Wilcoxon signed rank test. For cell proliferation, migration and invasion studies, statistical significance was analyzed by an unpaired $t$-test. $\mathrm{P}<0.05$ as considered to indicate a statistically significant difference. All calculations were performed using SPSS software (version 16.0; SPSS Inc., Chicago, IL, USA).

\section{Results}

JAG2 is overexpressed in CRC tissues and cell lines. IHC staining was used to assess the level and pattern of JAG2 protein expression in tissues from 40 cases of human CRC. JAG2 expression was detected in 38/40 CRC cases (95.0\%) and in 5/40 surrounding normal tissues (12.5\%), with a predominantly membrane/cytoplasmic localization (Fig. 1A and B). A 3.1-fold increase in median IHC score was observed in tumor regions compared to adjacent normal areas $(\mathrm{P}<0.0001)$ (Fig. 1C), indicating JAG2 overexpression in CRC tissues. In addition, JAG2 expression was detected by western blot in all

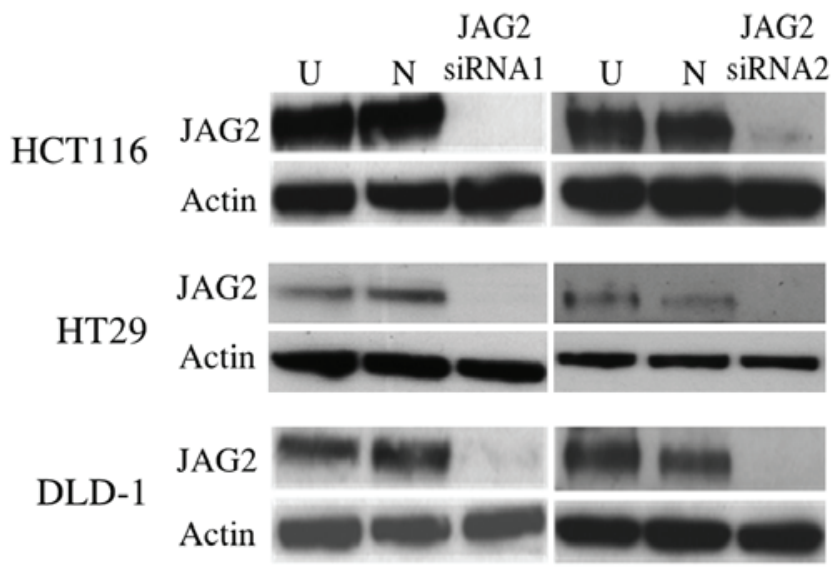

Figure 2. Western blotting results showing downregulation of JAG2 protein expression at $48 \mathrm{~h}$ post siRNA transfection in HCT116, HT29 and DLD-1 colorectal cancer cell lines. 'U' represents untransfected cells, 'N' represents cells transfected with negative control siRNA, and 'siRNA1' and 'siRNA2' represent cells transfected with two different kinds of JAG2 siRNAs. Actin was used as loading control. JAG2, Jagged 2; siRNA, small interfering RNA.

CRC cell lines tested, including RKO, HT29, HCT116, SW620, SW480 and DLD-1, but not in the non-malignant colon cell line, CCD-18Co (Fig. 1D). Together, these findings indicate that JAG2 protein is frequently overexpressed in CRC cells compared to non-malignant colon cells.

JAG2 silencing inhibits migration of CRC cell lines. To study the functions of JAG2 in CRC, RNA interference was used to knockdown its expression in three CRC cell lines: HCT116, DLD-1 and HT29. Two different siRNAs were used to provide support to the specificity of the observed effects. The functional consequences of JAG2 silencing on cell migration, 
A

U

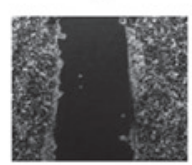

HCT116
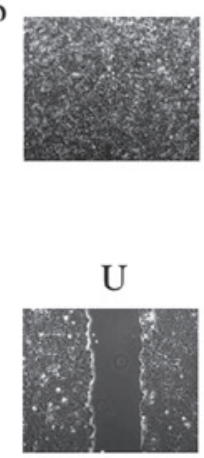

DLD-1

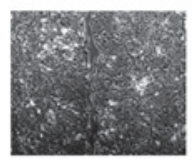

U

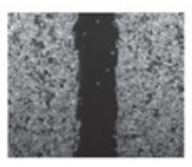

HT29

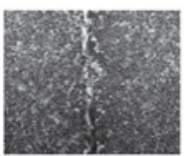

N
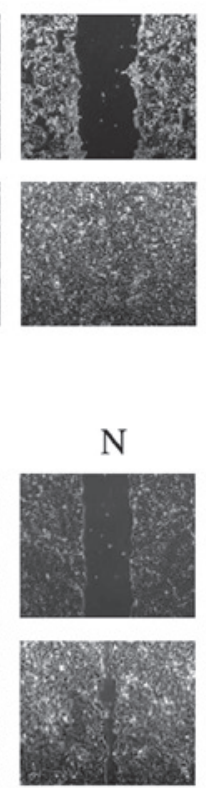

N
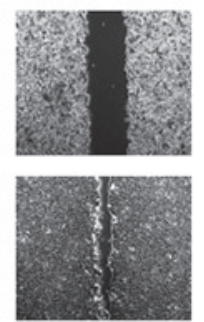

siRNA1
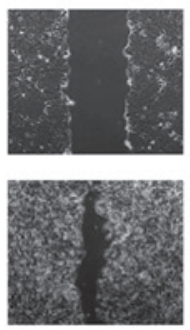

siRNA1
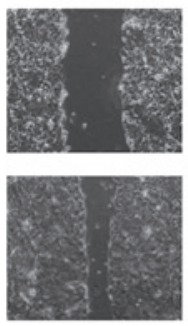

siRNA1
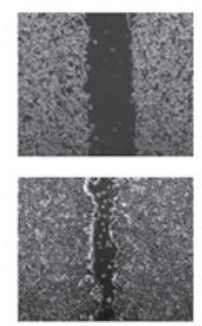

SiRNA2
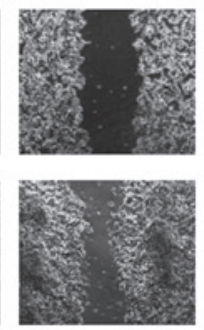

siRNA2
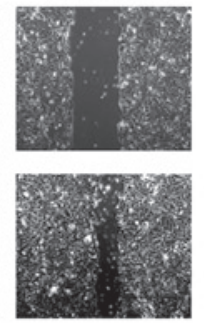

SiRNA2
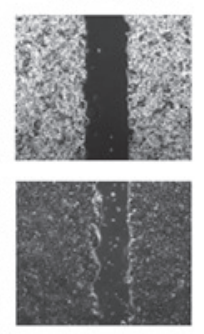

B

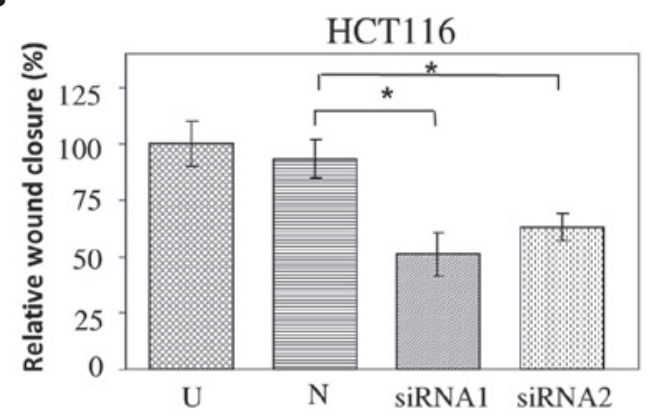

DLD-1
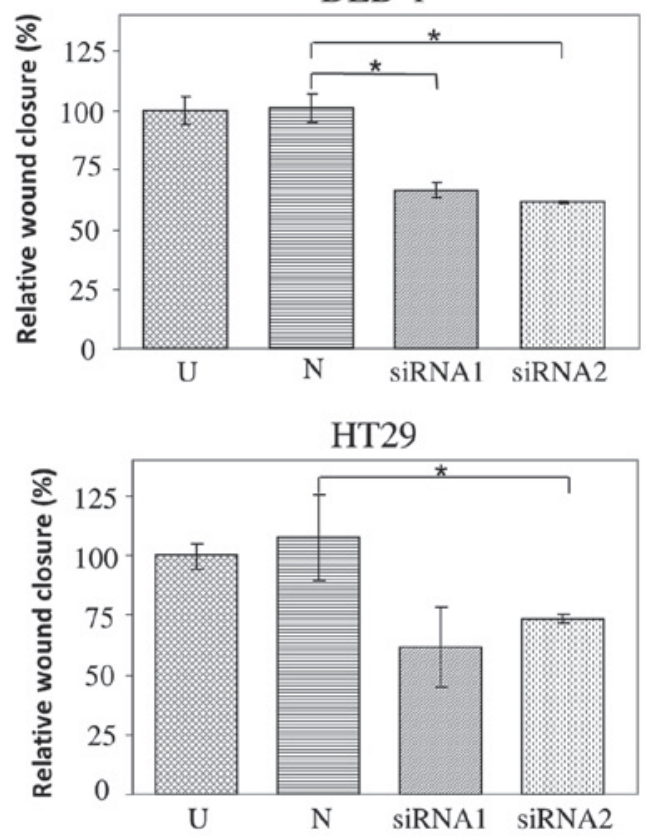

Figure 3. (A) Photos showing wound healing assay results at $72 \mathrm{~h}$ post siRNA transfection in HCT116, DLD-1 and HT29 CRC cell lines; JAG2 knockdown by two different siRNAs decreased motility of all three CRC cell lines (magnification, x50). (B) Bar charts quantifying the reduced migration of CRC cells following JAG2 silencing. Results are presented as the mean \pm standard error of the mean; 'P<0.05. ' $U$ ' represents untransfected cells, 'N' represents cells transfected with negative control siRNA, and 'siRNA1' and 'siRNA2' represent cells transfected with two different kinds of JAG2 siRNA. siRNA, small interfering RNA; CRC, colorectal cancer; JAG2, Jagged 2.

invasion and proliferation were then assessed by monolayer scratch wound healing, Matrigel invasion and cell growth assays, respectively.

JAG2 knockdown was confirmed at the protein level using western blot at $48 \mathrm{~h}$ post transfection (Fig. 2). Wound healing assays reveal impaired wound closure of cells transfected with either of the JAG2 siRNAs in HCT116, DLD-1 and HT29 cell lines compared to those transfected with negative control siRNA (HCT116, $\mathrm{P}=0.015$ for siRNA1 and $\mathrm{P}=0.018$ for siRNA2; DLD-1, $\mathrm{P}=0.005$ for siRNA1 and $\mathrm{P}=0.002$ for siRNA2; and HT29, $\mathrm{P}=0.082$ for siRNA1 and $\mathrm{P}=0.019$ for siRNA2) (Fig. 3A and B). This finding indicates that JAG2 silencing can inhibit the motility of the CRC cell lines tested.

JAG2 silencing inhibits the invasiveness of CRC cell lines. The effect of JAG2 silencing on cell invasion in HCT116 and DLD-1 cells was assessed using Matrigel invasion assays. HT29 cells do not invade through Matrigel and were thus excluded from this analysis. The findings demonstrate that the invasive capability of JAG2-silenced HCT116 and DLD-1 cells decreased significantly relative to cells transfected with negative control siRNA (HCT116, $\mathrm{P}=0.009$ for siRNA1 and $\mathrm{P}=0.021$ for siRNA2; and DLD-1, $\mathrm{P}=0.049$ for siRNA1 and $\mathrm{P}=0.013$ for siRNA2) (Fig. 4A and $\mathrm{B}$ ). This result indicates that JAG2 silencing is inhibitory for invasiveness of CRC cell lines and supports a pro-invasive role for JAG2 expression in CRC cells.

JAG2 silencing does not significantly affect cell proliferation in $C R C$ cell lines. The effect of JAG2 knockdown on cell proliferation was investigated. No significant difference in the number of HCT116, DLD-1 or HT29 cells was detected at $72 \mathrm{~h}$ post JAG2 knockdown compared with negative controls (HCT116, $\mathrm{P}=0.756$ for $\mathrm{siRNA} 1$ and $\mathrm{P}=0.196$ for siRNA2; DLD-1, $\mathrm{P}=0.265$ for siRNA1 and $\mathrm{P}=0.092$ for siRNA2; and HT29, $\mathrm{P}=0.583$ for siRNA1 and $\mathrm{P}=0.862$ for siRNA2) (Fig. 5), indicating that JAG2 knockdown does not significantly affect cell proliferation in all three CRC cell lines tested. Combined with the previously mentioned findings, this result indicates that JAG2 knockdown inhibits motility and invasiveness of CRC cell lines independently of mechanisms affecting cell proliferation. 
A



DLD-1

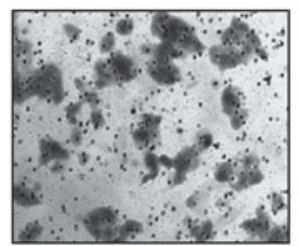

N
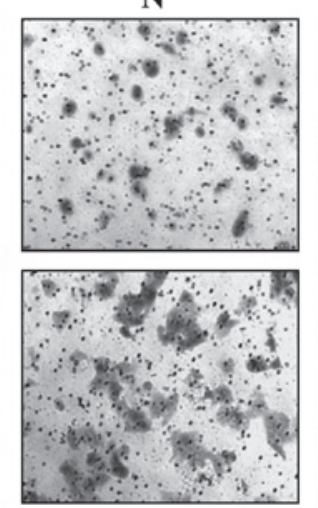

SiRNA1
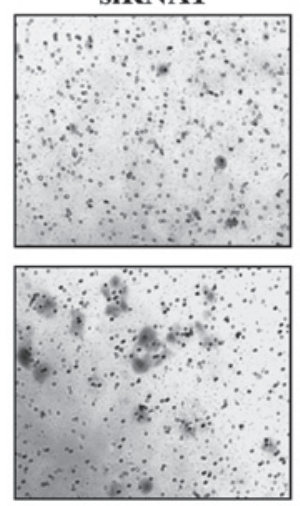

SiRNA2
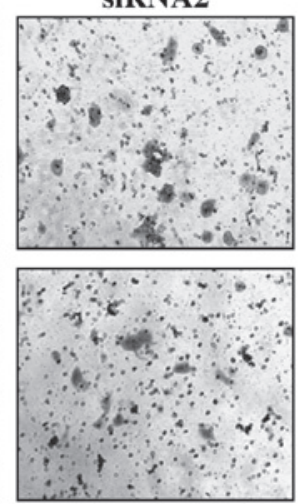

B

HCT116
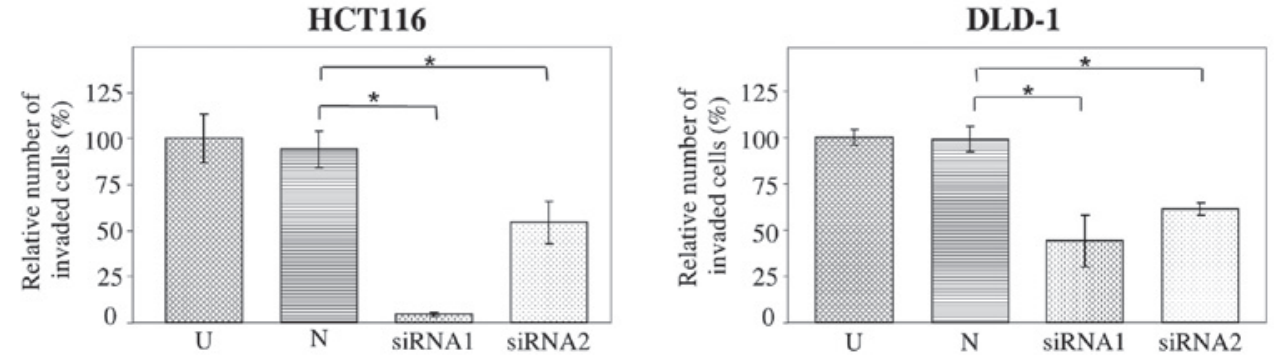

Figure 4. (A) Photos showing results from Matrigel invasion assays at $24 \mathrm{~h}$ post siRNA transfection; decreased invasiveness of HCT116 and DLD-1 cells was observed following JAG2 silencing by two different kinds of JAG2 siRNAs (magnification, x200). (B) Bar charts quantifying the reduced invasion of colorectal cancer cells following JAG2 silencing by two different kinds of siRNAs. Results are presented as the mean \pm standard error of the mean; ${ }^{*} \mathrm{P}<0.05$. 'U' represents untransfected cells, 'N' represents cells transfected with negative control siRNA, and 'siRNA1' and 'siRNA2' represent cells transfected with two different kinds of JAG2 siRNAs. siRNA, small interfering RNA; JAG2, Jagged 2.

\section{HCT116}

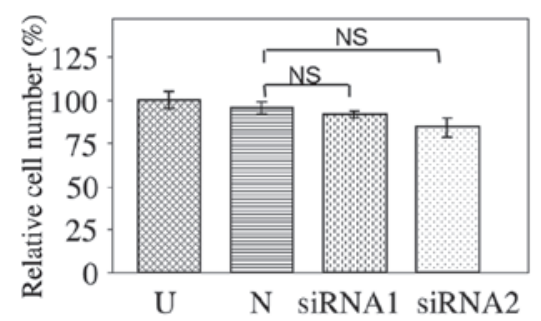

DLD-1

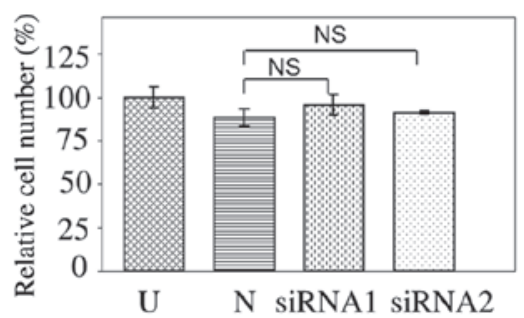

HT29

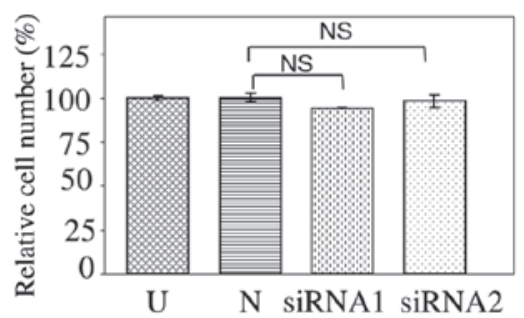

Figure 5. Bar charts quantifying relative cell numbers of HCT116, DLD-1 and HT29 cells at 72 h post siRNA transfection. JAG2 knockdown by two different kinds of JAG2 siRNA does not affect the proliferation of colorectal cancer cell lines. 'U' represents untransfected cells, 'N' represents cells transfected with negative control siRNA, 'siRNA1' and 'siRNA2' represent cells transfected with two different kinds of JAG2 siRNAs. Results are presented as the mean \pm standard error of the mean; NS indicates P>0.05. siRNA, small interfering RNA; JAG2, Jagged 2.

\section{Discussion}

Previous studies have indicated that Notch signaling is upregulated in CRC (15-18). However, data regarding the expression of JAG2 protein in CRC tissues is scarce. The current study provides insight on this topic by demonstrating that JAG2 expression is detected in up to $95 \%$ of CRC cases (38/40 patients) and is 3 -fold overexpressed in cancerous tissues compared with surrounding non-tumorous tissues. Furthermore, JAG2 expression was detected in all six of the CRC cell lines tested, but not in a normal colon epithelial cell line. These results are consistent with a previous study that demonstrated upregulated expression of JAG2 mRNA in all 20 CRC cases examined (15). Taken together, the finding that JAG2 is frequently overexpressed in CRC cells is suggestive of a crucial role for this protein in CRC development.
To gain a deeper understanding of the functional significance of JAG2 expression in CRC, the present study used RNA interference to investigate the effects of JAG2 knockdown in three CRC cell lines. The results indicated that JAG2 knockdown reduces migration in HCT116, DLD-1 and HT29 cells. It can also inhibit invasion of HCT116 and DLD-1 CRC cell lines. These effects were not mediated through a reduction in cell proliferation, as cell numbers were not significantly affected relative to cells transfected with negative control siRNA at $72 \mathrm{~h}$ post transfection. These findings implicate JAG2 expression in the promotion of CRC metastasis by increasing motility and invasiveness of CRC cells, and indicate that it may be involved in cancer progression rather than initiation.

A similar pro-metastatic function has been reported for JAG2 in other types of cancer. In breast cancer, JAG2 
expression was found to promote metastasis and was significantly associated with overall and metastasis-free survival of breast cancer patients $(11,19)$. Likewise, JAG2 was found to be capable of inducing the metastasis of lung adenocarcinoma cells in mice (20). These findings provide support to the hypothesis that JAG2 functions to promote metastasis in multiple cancer types. It would be of interest to investigate whether JAG2 is expressed in circulating tumor cells of CRC patients, as these cells have been demonstrated to be associated with tumor-node-metastasis stage and lymph node status $(21,22)$. In addition, the clinical value of JAG2 as a novel biomarker in CRC warrants further study (23).

In conclusion, the current study has demonstrated that JAG2 is overexpressed in a majority of CRC tissues and is involved in motility and invasiveness, but not proliferation, of CRC cells. Further studies are required to gain a deeper understanding of the precise mechanisms involved.

\section{Acknowledgements}

This work was supported by funding from the Chinese University of Hong Kong (Direct Grants 2041697, 4054004 and 4054005).

\section{References}

1. Torre LA, Bray F, Siegel RL, Ferlay J, Lortet-Tieulent J and Jemal A: Global cancer statistics, 2012. CA Cancer J Clin 2: 87-108, 2015.

2. McArdle C: ABC of colorectal cancer: Effectiveness of follow up. BMJ 321: 1332-1335, 2000

3. Golfinopoulos V, Salanti G, Pavlidis N and Ioannidis JP: Survival and disease-progression benefits with treatment regimens for advanced colorectal cancer: A meta-analysis. Lancet Oncol 8: 898-911, 2007.

4. Heinemann V, von Weikersthal LF, Decker T, Kiani A, Vehling-Kaiser U, Al-Batran SE, Heintges T, Lerchenmüller C, Kahl C, Seipelt G et al: FOLFIRI plus cetuximab versus FOLFIRI plus bevacizumab as first-line treatment for patients with metastatic colorectal cancer (FIRE-3): A randomised, open-label, phase 3 trial. Lancet Oncol 10: 1065-1075, 2014.

5. Katoh $\mathrm{M}$ and Katoh $\mathrm{M}$ : Notch signaling in gastrointestinal tract (review). Int J Oncol 30: 247-251, 2007.

6. Liu J, Sato C, Cerletti M and Wagers A: Notch signaling in the regulation of stem cell self-renewal and differentiation. Curr Top Dev Biol 92: 367-409, 2010.

7. Weng AP and Aster JC: Multiple niches for Notch in cancer: Context is everything. Curr Opin Genet Dev 14: 48-54, 2004.

8. Weng AP, Ferrando AA, Lee W, Morris JP IV, Silverman LB, Sanchez-Irizarry C, Blacklow SC, Look AT and Aster JC: Activating mutations of NOTCH1 in human T cell acute lymphoblastic leukemia. Science 306: 269-271, 2004.
9. Reedijk M, Odorcic S, Chang L, Zhang H, Miller N, McCready DR, Lockwood G and Egan SE: High-level coexpression of JAG1 and NOTCH1 is observed in human breast cancer and is associated with poor overall survival. Cancer Res 65: 8530-8537, 2005.

10. Pietras A, von Stedingk K, Lindgren D, Påhlman S and Axelson H: JAG2 induction in hypoxic tumor cells alters Notch signaling and enhances endothelial cell tube formation. Mol Cancer Res 9: 626-636, 2011.

11. Xing F, Okuda H, Watabe M, Kobayashi A, Pai SK, Liu W, Pandey PR, Fukuda K, Hirota S, Sugai T, et al: Hypoxia-induced Jagged 2 promotes breast cancer metastasis and self-renewal of cancer stem-like cells. Oncogene 30: 4075-4086, 2011.

12. Jung SG, Kwon YD, Song JA, Back MJ,Lee SY, Lee C, Hwang YY and An HJ: Prognostic significance of Notch 3 gene expression in ovarian serous carcinoma. Cancer Sci 101: 1977-1983, 2010.

13. Wong SC, Lo SF, Lee KC, Yam JW, Chan JK and Wendy Hsiao WL: Expression of frizzled-related protein and Wnt-signalling molecules in invasive human breast tumours. J Pathol 196: 145-153, 2002.

14. McLane MA, Zhang X, Tian J, Zelinskas C, Srivastava A, Hensley B and Paquette-Straub C: Scratching below the surface: Wound healing and alanine mutagenesis provide unique insights into interactions between eristostatin, platelets and melanoma cells. Pathophysiol Haemost Thromb 34: 164-168, 2005.

15. Reedijk M, Odorcic S, Zhang H, Chetty R, Tennert C, Dickson BC, Lockwood G, Gallinger S and Egan SE: Activation of Notch signaling in human colon adenocarcinoma. Int $\mathrm{J}$ Oncol 33: 1223-1229, 2008.

16. Meng RD, Shelton CC, Li YM, Chetty R, Tennert C, Dickson BC, Lockwood G, Gallinger S and Egan SE: gamma-Secretase inhibitors abrogate oxaliplatin-induced activation of the Notch-1 signaling pathway in colon cancer cells resulting in enhanced chemosensitivity. Cancer Res 69: 573-582, 2009.

17. Peignon G, Durand A, Cacheux W, Ayrault O, Terris B Laurent-Puig P, Shroyer NF, Van Seuningen I, Honjo T, Perret C and Romagnolo B: Complex interplay between $\beta$-catenin signalling and Notch effectors in intestinal tumorigenesis. Gut 60: 166-176, 2011.

18. Serafin V, Persano L, Moserle L, Esposito G, Ghisi M, Curtarello M, Bonanno L, Masiero M, Ribatti D, Stürzl M, et al: Notch3 signalling promotes tumour growth in colorectal cancer. J Pathol 224: 448-460, 2011.

19. Nam DH, Jeon HM, Kim S, Kim MH, Lee YJ, Lee MS, Kim H, Joo KM, Lee DS, Price JE, et al: Activation of notch signaling in a xenograft model of brain metastasis. Clin Cancer Res 14: 4059-4066, 2008.

20. Yang Y, Ahn YH, Gibbons DL, Zang Y, Lin W, Thilaganathan N, Alvarez CA, Moreira DC, Creighton CJ, Gregory PA, et al: The Notch ligand Jagged2 promotes lung adenocarcinoma metastasis through a miR-200-dependent pathway in mice. J Clin Invest 121: 1373-1385, 2011.

21. Wong SC, Chan CM, Ma BB, Hui EP, Ng SS, Lai PB, Cheung MT, Lo ES, Chan AK, Lam MY, et al: Clinical significance of cytokeratin 20-positive circulating tumor cells detected by a refined immunomagnetic enrichment assay in colorectal cancer patients. Clin Cancer Res 15: 1005-1012, 2009.

22. Wong SC, Ng SS, Cheung MT, Luk LY, Chan CM, Cheung AH, Lee VH, Lai PB, Ma BB, Hui EP, et al: Clinical significance of CDX2-positive circulating tumour cells in colorectal cancer patients. Br J Cancer 104: 1000-1006, 2011.

23. Wong SC, Chan CM, Ma BB, Lam MY, Choi GC, Au TC, Chan AS and Chan AT: Advanced proteomic technologies for cancer biomarker discovery. Expert Rev Proteomics 6: 123-134, 2009. 